\title{
15
}

\section{A Teamwork Approach to Decision Support System Development for Watershed Management Problems}

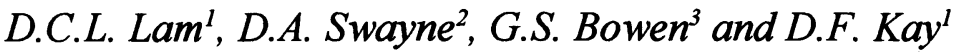

${ }^{1}$ National Water Research Institute, Environment Canada, Burlington, Ontario, CANADA L7R 4A6, ph. 905-336-4916, fax 905-336-4582 email:David.Lam@cciw.ca and Doug.Kay@cciw.ca ${ }^{2}$ Dept. of Computing and Information Sci., U. of Guelph, Guelph, Ontario, CANADA NIG 2WI email: dswayne@snowhite.cis.uoguelph.ca ${ }^{3}$ Watershed Management Section, Ontario Ministry of Environment and Energy, Toronto, Ontario,CANADA M4V1L5 email:boweng@gov.on.ca

\begin{abstract}
Decision-support systems (DSS) for solving complex environmental problems are not merely computer software systems. A working system requires data and knowledge inputs from domain scientists and the results need to be easily understandable to decision makers. It must be designed with both scientists and end users in mind, and must involve them early in the development, all working as a team. They need to overcome attitude and communication problems that exist among them and the system. This paper discusses the issues surrounding a team approach of building a DSS.
\end{abstract}

\section{Keywords}

Decision support system, knowledge-based system, environmental information system

\section{INTRODUCTION}

Environmental problems such as climate change and watershed management are multidisciplinary in nature. Solutions require the understanding of many disciplines of science: meteorology, hydrology, geochemistry and ecology, etc. With the advent of the computer, scientific data are now 
readily stored in computer databases and can be retrieved with sophisticated structured query languages (SQL), sometimes through fast computer network. The knowledge of the scientific processes, e.g. atmospheric transport or nutrient dynamics, are often encapsulated in computer simulation models.

As computer applications in data- and knowledge-bases increase, one would expect that the linkage between data and models is made more user-friendly. Significant efforts have been made (e.g. Loucks and da Costa, 1991) to promote the development of so-called decision-support systems (DSS) which can help, if not literally deliver the data and models to the hands of the decisionmakers, at least demonstrate how the gap between science and policy could be brought closer. However, several obstacles remain in the development and operation of these decision support systems.

At the outset, the problem is not so much with the databases. It is always possible, given time, to convert from one data format to another. Yet, scientists and decision-makers still experience the so-called "information gridlock" syndrome. For as long as different agencies continue to collect environmental data, without coordination, it is difficult to find out how much data there are and who owns the data. Sometimes, the real hardship is to obtain the consent to use the data. Similar institutional problems exist with the use of models. It is often difficult for the designer of the decision-support system to predetermine which models to use. There are simply too many models to choose from, or scientists may offer their own favourite models instead of pre-selected ones. Thus, if the system is designed without these team considerations and the flexibility to accommodate "team" data and models, it will face strong resistance or "attitude" problems eventually.

\section{THE RATIONALE AND THE APPROACH}

One may challenge the notion that an open or teamwork-based DSS framework can actually change one's attitude. Our experiences over the past ten years show that DSS is basically a communication vehicle between scientists and policy makers, with the DSS developer in the middle. Typically one would start with the policy makers or the users to understand the problem to be solved and their expectation of how DSS may provide them with solutions. Then to bring in the data and models, one may need to talk to scientists familiar with the problem, since the DSS developer is usually not a domain expert. When relevant data and models are blended in with necessary supporting tools such as Geographical Information Systems (GIS), graphical interfaces and models, the prototype is to be tested by the user. More problems and requirements are then identified and the final product would be polished through several cycles of iterations with the scientists and the policy advisors.

Most scientists and policy advisors are cooperative since it is in their best interest to work toward a successful final product. However, since DSS development is about communication among people with diverse backgrounds, it is unavoidable that the communication may at times slow down because of lack of understanding and trust. For example, there was the tendency to hold back data, information or knowledge because the intent of the DSS was not explained clearly or scientists are afraid that their data and knowledge might be misused. Some believed that combining data and 
models from different disciplines would not generate new knowledge and therefore the DSS exercise was redundant. Some believed that they should be the one to be consulted directly by policy advisors, for fear that DSS could distort their knowledge if unsuccessful and could displace them if successful. Decision-makers or advisors, on the other hand, were generally concerned about whether there might be good, credible science implemented in the system. They were concerned about the cost, the socio-economical impact and the policy aspects of the decision. They were not interested in too much scientific details, but required a clear answer with limited quantifiers on uncertainties.

Given that the DSS is a team exercise, we have to deal with these human communication problems at the outset. Our experience is that the sooner we can build a prototype, the better we could use it to communicate among team members. Using examples, we can explain better the purpose and functionality of system collectively being built. We can then quickly overcome the fear and mistrust, if any, among the experts. We also provide the policy advisors a glimpse of what the DSS can do and let them provide an early input into the design of the system. The disadvantage is that the data and models may be too preliminary and the results too crude. However, we can turn this disadvantage into an advantage by showing the need for better data and models and challenging the experts to provide them. Indeed, we were able to obtain their data and models more effectively this way.

Thus, our approach is to develop a framework containing generic modules that are already tightly linked and allow information flow freely among the database, models, GIS, computer graphics and expert systems (Lam and Swayne, 1991). Recently, we have expanded the framework under the Windows system. By making use of advanced features in Windows, we are able to open more freely the connection to other databases and models. The user of such a framework can easily put their own data and models in the system and automatically linked up with those from other team members, without having to construct the linkages themselves. In this way, the team can devote more time with the problem than to struggling with information technologies.

\section{THE FRAMEWORK}

The RAISON system (Lam and Swayne, 1991) under the Windows environment is designed with this teamwork approach in mind. The system can be used to integrate environmental data for air, water, soil, sediments and biota or to couple models for these media so that scenarios for management options and cost optimization can be visualized with computer graphics and maps (Lam et al., 1994). The system offers generic modules already linked to each other for manipulation of databases, models, graphics, GIS and optimization procedures, etc.

Figure 1 shows the framework for solving an environmental problem such as watershed management. Typically, information for each of the disciplines such as socio-economics, air issues, land, water, ecology and human health can be entered to the system and linked up through the database and GIS. For example, for watershed management problems, the industry may produce air pollutants or agricultural activities may lead to dispersion of pesticides, which may enter the 
terrestrial and aquatic regimes, thereby endangering fish species. The hydrological pathways can be modelled and the computed runoff can be used in chemical transport models to determine the ecological impact, etc. The ecological impact or damage cost can be used as the objective function in the overall optimization procedure (Fig. 1) that would select the best policy strategies (regulating water flows, expanding sewage treatment, farming practices, etc.) to achieve the preset goal. As well, the uncertainty estimation and error propagation for the connected models can also be determined to become part of the advice to the policy makers on how much confidence level that can be placed on the model predictions (Fig. 1).

\section{WATERSHED MANAGEMENT}

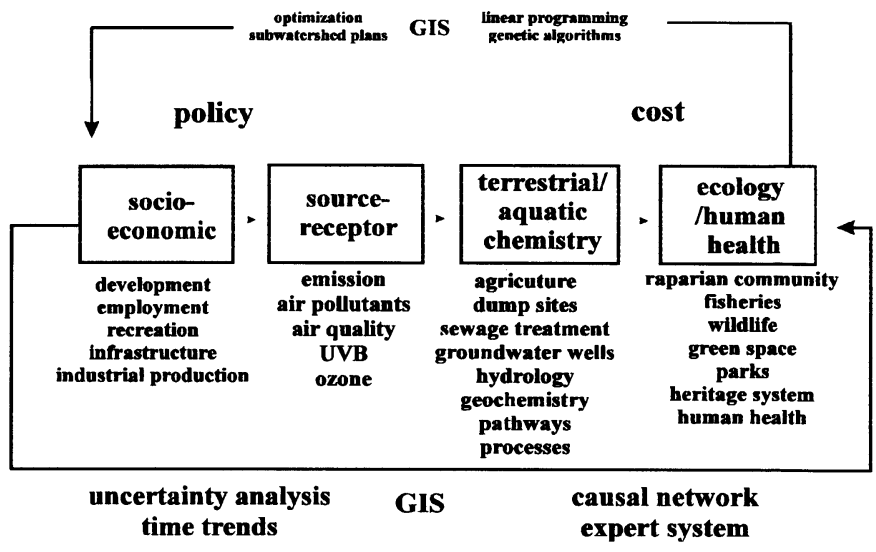

Figure 1 A framework for a decision support system for watershed management.

\section{INTEGRATION OF DATA AND OBJECTS}

\subsection{Software design considerations}

Data can be entered to the RAISON/Windows system through conversion interfaces available for many databases in the Windows system. GIS maps can be entered in vector and raster formats and can be used in overlay analysis of different map layers of information. For example, in Figure 2, information on fish can be retrieved from the database for a watershed and can be displayed against different background maps, e.g. stream and roads or geology (Fig. 2), from different sources. As expected, the trout species tend to thrive better in upstream locations because they prefer colder temperature, whereas a cursory, visual comparison of fish abundance data and geological factors (Fig.2) can lead to further study. It is this type of quick data visualization that helps promote new ideas and find new knowledge not easily attainable without the aid of such an integrated system. 


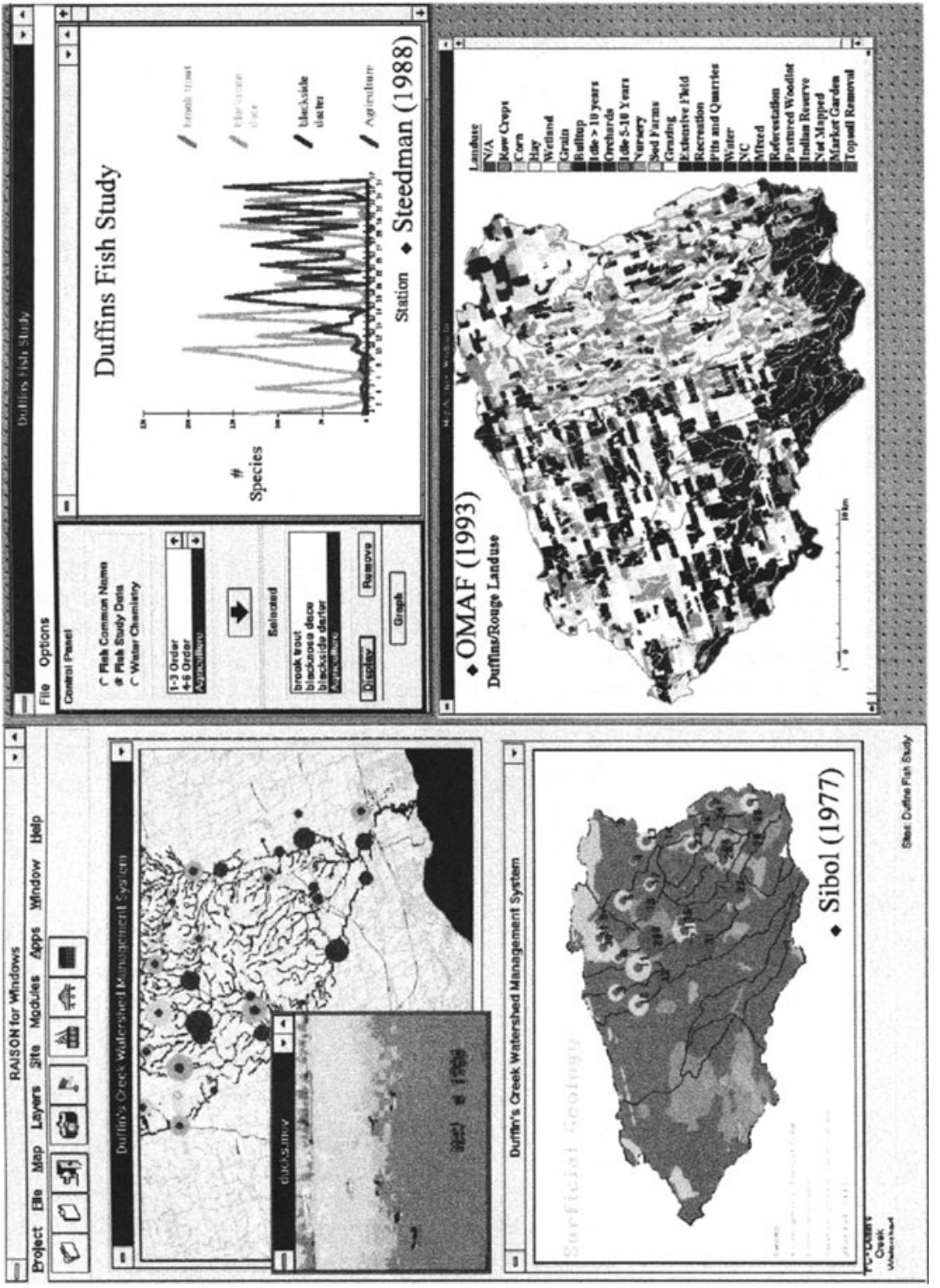

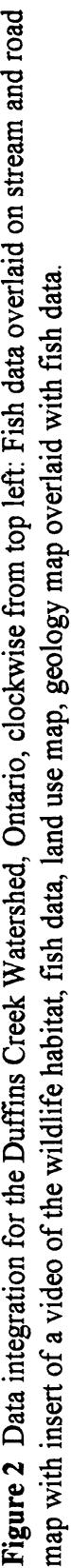


Similarly, maps on soil types and land use (Fig. 2) can be used to derive model coefficients for various types of hydrological models. In the RAISON system, special functions are developed and made available through a library of functions to automate algorithms for deriving model coefficients from GIS maps for these models. These GIS functions are useful for deriving other generic characteristics, e.g. slopes and flow directions, required by other models. Other information such as textual description of data or a video recording the movement and sounds of wildlife in their natural habitat (Fig. 2) can be accessed by selecting the appropriate sampling station. These video and items are stored as objects using the object-embedding and object-oriented programming techniques under the Windows environment. Thus, the objects can be called up via a relational database, by attached a dynamic linkage to a map icon, or through the execution of an expert system which activates selected objects based on model results. This technique of integrating data and objects serves well in bridging the gap between complex environmental data and simple pictures and images.

\subsection{Team work considerations}

From our experiences, it is important to show the identity of the data source once the data are made available in the DSS. Not only proper credits and acknowledgements are provided, but also contact addresses are required to facilitate communication among providers and receivers. An annotation window associated with each database is always useful to highlight the essential information such as the analytical chemistry method or the interpolation scheme used, the measurement units, the field conditions during data collection, and limitations in the use of the data. The system should provide the facility to bring in new data sets quickly, sometimes almost instantaneously during team workshops, among team members who may bring their own data in electronic forms or via local/wide area computer networks. From our experiences, the Windows environment allows many software products for database and spreadsheets to transfer data into a system such as RAISON easily. In many instances, the transfer is only temporary, via the Windows Clipboard into RAISON, so that team members can still use their own database or spreadsheet software to illustrate their data and use the RAISON system for connecting to other databases, maps or models. In this way, we provide a wider margin of comfort to those team members who prefer to use their own database systems and familiar routines, while offering the opportunity to integrate their data with others in RAISON. We find this approach quite effective in helping new team members overcome the initial institutional barrier. As they become more familiar with the team work approach and the RAISON system, they generally see the benefits of working together to solve practical problems, and then the data flow and accessibility are no longer temporary but more committed to the team.

\section{INTEGRATION OF MODELS AND MANAGEMENT NEEDS}

\subsection{Software design considerations}

The DSS framework provides an open structure for allowing different types of models to be linked with each other. For example, for nonpoint source pollution, a hydrological model is used to compute surface runoff which is then used as input to an agriculture nutrient/pesticide model. The 
nonpoint source inputs of nutrients and toxics are then linked to an advection-dispersion model that can also handle point source problems such as sewage effluent (Fig. 3). Further linkage to groundwater pollution models is possible. Under the DSS framework (Fig.1), these models can be incorporated into the system by: (a) introducing the source code as given or rewritten in a programming language compatible with $\mathrm{C}, \mathrm{C}++$ or Visual Basic and compiling it as part of the system executable, (b) using simple programming tools to develop an interface that intercepts the input and output data of the model into the DSS, but using only the executable code of the model (i.e. no need to rewrite the models), or (c) executing the model essentially on its own using an input file created a priori, saving the output in a database and then reading the output files from the DSS. The interaction of the model with the DSS is the greatest in (a) but the programming effort is correspondingly the highest. The opposite is (c) where both the interaction with DSS and the programming effort are the least. From our experience, since method (b) offers the compromise between DSS interaction and programming effort, we used it in most cases. Alternatively, if the model inputs and outputs themselves are sufficient to support the creation of a simple input-output model, we can use the input-output model so derived to emulate the original model and, if possible, to use it in the DSS for the specific application. The results may be approximations but the DSS interaction would be the fastest, even faster than the original model.

The need to have faster interaction with other models in DSS is obvious when management strategies are considered. For example, when designing the flowpath and reservoir network, or when establishing the cost of expanding an existing sewage treatment plant to achieve a downstream water quality standard, the models will be run hundreds of times as they are nested in an optimization procedure (Fig. 3). For engineering design problems, such rigorous investigation is required. For some management consideration, however, particularly when the objectives, e.g. ecological impact, are fuzzy and the cost estimates are crude, less rigorous answers may be acceptable. In the latter case, the use of artificial intelligence techniques such as genetic algorithm, fuzzy expert system and causal network (Lam and Swayne, 1994) can offer reasonable iteration rates and are flexible for generating practical answers without greatly compromising the science. In the end, what the scientific and socio-economic team can deliver to the decision-makers is a system composing of their joint data- and knowledge-bases with only a few menu buttons for displaying simple results and recommendations, including the advice on decision and approval relating to possible actions, e.g. sewage treatment plant expansion (Fig. 3).

\subsection{Team work considerations}

For the benefit of the team, it is important to identify the source of the models and to provide an annotation of their assumptions, limitations and applicability, once they are incorporated as part of the DSS. Generally, modellers tend to promote their own favourite models, either by virtue of being the authors themselves or simply being more familiar through practice. The institutional barrier is therefore great when one modeller claims to have a better model than others, often arguing over whose model has a better process formulation, a finer scale resolution, or a more accurate computational method. However, while these are basic elements for constructing a model, the choice of the most appropriate model for solving a practical problem often depends on the questions being asked (Lam and Swayne, 1991). For as long as the model can answer the questions within 


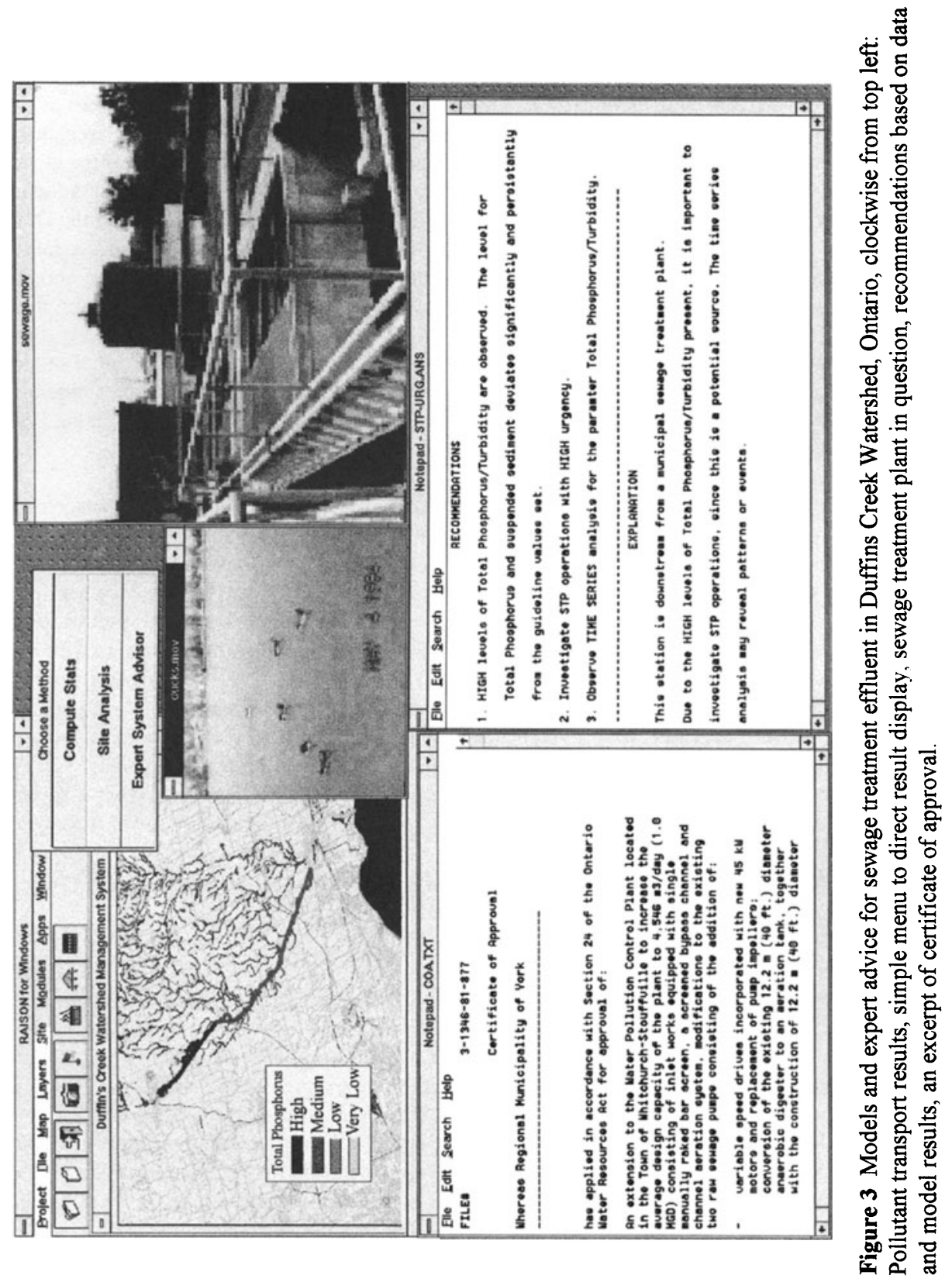


a reasonable tolerance on the accuracy of assumptions, scales and methods, it is a good model. A good model can be applicable in an integrated assessment only when it fits within the tolerance of, and is capable to link to, models selected likewise from other disciplines. In other words, not only do the modellers have to learn models developed in their own and other disciplines, but also they have to accept the integrated assessment perspective in determining which model is more appropriate. To evercome this barrier, our experiences show that, by and large, when the assumptions, scales and methodologies of the models, regardless of their original disciplines, are presented, a short list of eligible models emerges. To select the most appropriate among these eligible models, an expert system is used, first by going through the assumptions and applicability of the models and then building a rule base for selecting models based on available data and knowledge, with the full participation of the modellers and other experts in the team. Thus, the rule base is transparent and understandable to everyone, and the models are chosen based on team advice and available information. Similar team considerations are needed in selecting constraints and criteria for the optimization of resources and scenario testing for management strategies, although the rules are probably more uncertain and fuzzier than the case of model selection.

\section{CONCLUSION}

Over the past ten years, we have developed a generic DSS framework to help solve a wide range of environmental problems, from acid rain and mine effluent to watershed management and climate change. What impressed us most during these ten years are not so much the rapid advances in information technologies. It is the day-to-day attitude problem among team members and the communication problem between human and the machine. If these team building problems are ignored, the DSS remains an empty shell. Future information technology development should therefore be directed to facilitate this team building exercise: the assessibility of databases and maps through fast networks, the better documentation and linkage among models and the "soft" engineering tools that allows qualitative knowledge to be formally and critically used alongside with models and other decision support methodologies.

Acknowledgements. The authors thank E.D. Ongley and P. Miller for their support and the research partners in this project for generously providing data and knowledge.

\section{REFERENCES}

Lam, D.C.L. and Swayne, D.A. (1991) Integrating database, spreadsheet, graphics, GIS, statistics, simulation models and expert systems: experiences with the RAISON system on microcomputers, in Decision Support Systems - Water Resources Planning (ed. Loucks and da Costa), Springer-Verlag publ., Berlin, p. 429-460.

Lam, D.C.L. and Swayne, D.A. (1994) A hybrid expert system and neural network approach for environmental applications. Proc. 2nd Int. Conf. Expert Systems for Development, (Ed. R. Sadananda), IEEE Computer Society Press, Los Alamitos, California, p. 298-303. 
Lam, D.C.L., Mayfield, C.I., Swayne, D.A. and Hopkins, K. 1994. A prototype information system for watershed management and planning, J. Biological Systems, 2 (4), 499-517.

Loucks, D.P. and da Costa, J.R. 1991. Decision Support Systems - Water Resources Planning, Springer-Verlag Publ., Berlin, 574 pp.

\section{BIOGRAPHY}

David C.L. Lam received a Ph. D. in Computer Science from the University of Waterloo in 1974. He is currently a Senior Scientist and Project Chief, Environmental Information Technology, New Technologies Research Branch at the National Water Research Institute of Environment Canada. He was also a part-time Professor in the Civil Engineering Department, MacMaster University, and is currently an Adjunct Professor in the Computer Science Department, University of Waterloo, Canada. His research interests are in environmental modelling, "soft" engineering, decision support system and environmental information system.

David A. Swayne received a Ph.D. in Computer Science from the University of Waterloo in 1975. $\mathrm{He}$ is a member of the Society for Industrial and Applied Mathematics. He is currently a Professor in the Computing and Information Science Department and the School of Engineering at the University of Guelph, Canada. He is also an Adjunct Professor in the Computer Science Department at the University of Waterloo. Originally a specialist in numerical software, his research has focussed since 1983 on applications of computing science and information technology to the environment.

Gary S. Bowen has a B. Sc. degree from Trent University, Canada, and is currently a watershed management specialist with the Watershed management Section, Science and Technology Branch, Ontario Ministry of the Environment and Energy.

Douglas F. Kay has a B. Sc. degree from University of Guelph and is currently a Physcial Scientist with the New Technologies Research Branch, National Water Research Institute, Environment Canada. He specializes in data visualization and ecological systems. 